\title{
Impact of TFET Reverse Currents Into Circuit Operation: A Case Study
}

\author{
Juan Núñez \\ Instituto de Microelectrónica de Sevilla, IMSE-CNM (CSIC/Universidad de Sevilla) \\ Av. Américo Vespucio s/n, 41092, Sevilla (Spain) \\ e-mail: jnunez@imse-cnm.csic.es
}

\begin{abstract}
Tunnel FET transistors (TFETs) are one of the most promising candidates to replace CMOS transistors for future integrated circuits. However TFET-based circuit design can exhibit significant limitations due to their reverse conduction currents caused by the direct bias of the intrinsic diode of these transistors. In this paper we analyze in depth this issue through the design of charge pump (DC-DC step up converters) circuits for energy harvesting applications. The proposed solution mitigates the impact of reverse conduction currents and, thus, improves power conversion efficiencies (PCE) compared to previous designs.
\end{abstract}

Keywords - Tunnel transistors, Steep subthreshold slope, Charge pump, Reverse conduction, Energy harvesting.

\section{INTRODUCTION}

Portable and wearable devices for Internet of Things (IoT) applications rely on batteries whose lifetime could be extended by harvesting environmental energy sources. Moreover, for devices with low enough power requirements and expensive battery replacement, the battery may be dispensed with and rely exclusively on those harvesting ambient energy sources. This scenario, where ultra-low power devices and systems are combined with energy harvesting, has led to the emergence of new applications that previously were not possible. Emerging technologies, including emerging transistor devices, circuits, and architectures, allow aggressive scaling of supply voltages to achieve ultra-low power operation and, thus, overcome power density and energy efficiency challenges exhibited by CMOS technologies [1].

Tunnel field-effect transistors (TFETs) are one of the most attractive steep subthreshold slope $(S S)$ devices currently being investigated as a means of overcoming such limitations of CMOS technology [2]-[4]. A smaller SS makes it possible to lower threshold voltage while keeping leakage current under control, facilitating low voltage operation. The TFET is a gated reverse-biased $p-i-n$ diode with asymmetrical drain/source doping profiles $\left(\mathrm{n}^{+} / \mathrm{p}^{+}\right.$for the $N$-TFET and $\mathrm{p}^{+} / \mathrm{n}^{+}$for $P$-TFET). The operation of a TFET is based on the band-to-band tunneling (BTBT) of carriers from the valence band to the conduction band through the forbidden bandgap or vice versa. When the gate voltage is close to zero the TFET is in the off-state. When a positive (negative) enough gate voltage is applied to an $N$ type ( $P$-type) TFET, electrons (holes) in the valence (conduction) band in the source can tunnel to the conduction (valence) band in the channel.

Unlike MOSFETs, TFETs exhibit conduction only in the forward-bias region $\left(V_{D S}>0\right.$ for $N$-TFET, $V_{D S}<0$ for $P$ TFET). When the TFET is reverse-biased, under moderate bias conditions, minimal conduction currents are observed. For high-reverse bias voltages, significant negative currents are obtained regardless the gate voltage. Fig 1 depicts the
$I_{D S}-V_{D S}$ curves of an $N$-TFET for different $V_{G S}$ values (positive and negative). Note that under enough negative $V_{D S}$, the forward biased $p-i-n$ diode of the TFET conducts a large current. The simulated TFET is a $20 \mathrm{~nm} \mathrm{GaN/InN}$ single gate TFET [5]. The current model, based on the Kane-Sze formula for tunnelling, is valid in all four operating quadrants of the TFET. It uses a simple analytic model of the gate drain capacitance. Model parameters derived for different TFET structures showed good agreement with atomistic/TCAD device simulations. $P$ TFETs assume identical drive-on currents and capacitances. Models have been obtained from the nanoHUB website [6]. Circuits for logic applications have been evaluated using TFETs, showing power benefits for iso-performance or higher performance at iso-power up to moderate operating frequencies. However, benefits in higher performance domains in power or thermal limited applications could be achieved [7]-[9]. Recently, other applications domains in addition to logic ones have been identified.

In particular, and relevant to the IoT application field, it has been shown that front-end circuits for energy harvesting can benefit from the superior performance of TFETs at low voltages [10], [11]. High sensitivity RF to DC passive rectifiers and DC-DC step up charge pumps exhibiting much higher power conversion efficiency $(P C E)$ al low input power than $\mathrm{Si}$ FinFETs counterparts have been demonstrated [12]-[16]. These TFET-based circuits take advantage of the reduced turn-on voltage of these transistors with respect to $\mathrm{Si}$ FinFET to very much improve performance with weaker input signals. In addition, the higher $I_{O N}$ current exhibited by TFETs at low supply voltages translates into a reduced on-state resistance which contributes to decrease resistive power losses improving $P C E$. Finally, according also to [12]-[16], unidirectional conduction of TFETs has a positive impact on PCE through reduced reverse losses. Reverse losses are associated to the symmetric conduction of conventional transistors which conduct under both positive and negative drain to source voltages. However, although $P(N)$ TFETs do not conduct under low positive (negative) drain to source voltages, enough high reversed drain to source voltages produce the forward biasing of their intrinsic $p-i-n$ diode which translates in large losses currents. That is, the advantage associated to unidirectional conduction is limited to enough low amplitude input signals (or enough low input power). In this paper, we analyze in depth the operation limits of TFET-based switched capacitor charge pumps $(S C C P)$ with respect to voltage levels of input signals and input power, and propose tuning the $S C C P$ topology to the specific TFET characteristic to better control reverse currents and thus, extend the voltage/power operation range of TFET-based charge pumps. 
The rest of the paper is structured as follows. In Section II a simple SCCP is used to illustrate the limitations of its TFET-based implementation due to the forward biasing of the $p-i-n$ diode. The modification of the original topology is introduced. Simulation results are presented in Section III and conclusions are drawn in Section IV.

\section{DESIGN CHALLENGES OF TFET-BASED CHARGE PUMPS}

This section discusses relevant aspects of the design of TFET-based SCCP circuits. Fig 2a shows the schematic of the selected series-parallel SCCP [17]. Although most efficient topologies could be selected, its simplicity will clearly illustrate the inherent problem of the design of charge pumps using TFETs.

When the switches are in the position depicted in the figure, capacitors $C_{1}$ and $C_{2}$ are simultaneously charged to the same voltage $\left(V_{I N}\right)$ in parallel (hereinafter "parallel phase", $P A R)$. For the other switch configuration capacitors are connected in series, resulting in an output (ideally) double the input voltage (hereinafter "series phase", SER). Fig $2 \mathrm{~b}$ depicts the schematic of the circuit when the switches are replaced by transistors which are controlled by a gate voltage $V_{C L K}$. The correspondence between switches, transistors and the phases in which they are activated is shown in the inset table of Fig 2a. Currents flowing through each capacitor and transistor have been marked in the figure (positive currents in the direction of the arrows).

The ideal operation of the circuit is described below. When the clock signal is high ( $P A R$ phase), transistors $M_{l}, M_{3}$ and $\mathrm{M}_{4}$ are on, charging both capacitors to the input voltage. Transistors $\mathrm{M}_{2}$ and $\mathrm{M}_{5}$ are off. Therefore, the current through capacitor $C_{l}$ is equal to that of $M_{l}$ and that flowing through capacitor $C_{2}$ is equal to that of $M_{3}$ and $M_{4}$. Subsequently, when the clock signal switches low and the $S E R$ phase is activated, only the transistors $M_{2}$ and $M_{5}$ are switched on. As shown in Fig 2c, at the beginning of the $S E R$ phase the activation of the transistor $M_{2}$ allows the voltage between the two capacitances $\left(V_{C 2, N}\right)$ to take the value with which the capacitance $C_{1}$ was charged in the $P A R$ phase $\left(V_{I N}\right)$. Since $C_{2}$ was also charged with a $V_{I N}$ voltage in the $P A R$ phase, the voltage $V_{C 2, P}$ equals twice the input voltage. Thus, the series connection of both capacitors allows the output impedance to be charged to a value equal to twice the input voltage.

However, the design of these circuits using TFETs is not as straightforward because it must take into account the particular characteristics of these transistors in terms of their unidirectionality. As discussed in the previous section, the TFET could exhibit significant reverse currents for very negative (positive) values of the drain-source voltage of $N$ $(P)$ transistor. For this topology, transistor $M_{5}$ could discharge capacitor $C_{L}$ in the $P A R$ phase since $V_{D S, M S}=V_{I N}$ and, therefore, being $I_{M S} \neq 0$ in that phase. Similarly, in the $S E R$ phase, $M_{4}$ could have reverse conduction currents for high values of the input voltage since $V_{D S, M 4}=-V_{I N}$. In this scenario, part of the current flowing through the series connection of capacitors $C_{1}$ and $C_{2}$ would go through $M_{4}$ instead of charging the output impedance via $M_{5}$. Fig $2 \mathrm{~d}$ illustrates this idea by depicting simulation results for currents $I_{M 4}$ and $I_{M 5}$ for $V_{I N}=0.2 \mathrm{~V}$ (black) and $V_{I N}=1 \mathrm{~V}$ (gray). Note that significant reverse currents (negative currents in the figure) are observed for the largest value of the input voltage because drain-to-source voltages of

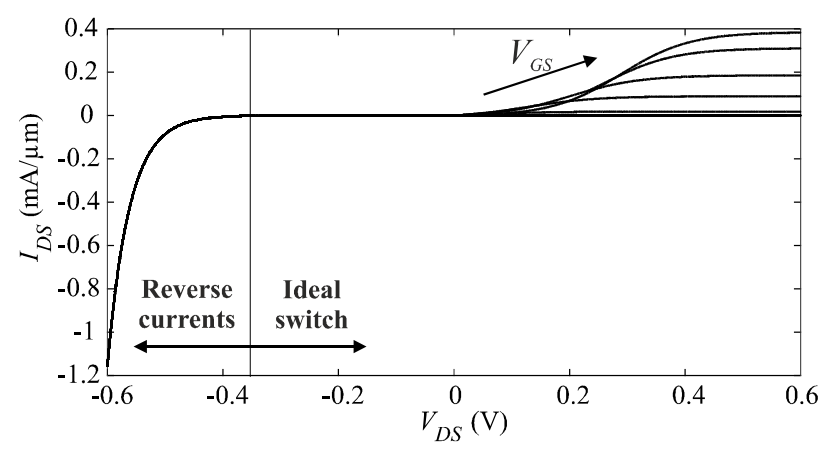

Fig 1. $I_{D S}-V_{D S}$ for N-TFET transistor reported in [5] for positive and negative $V_{G S}$ values.

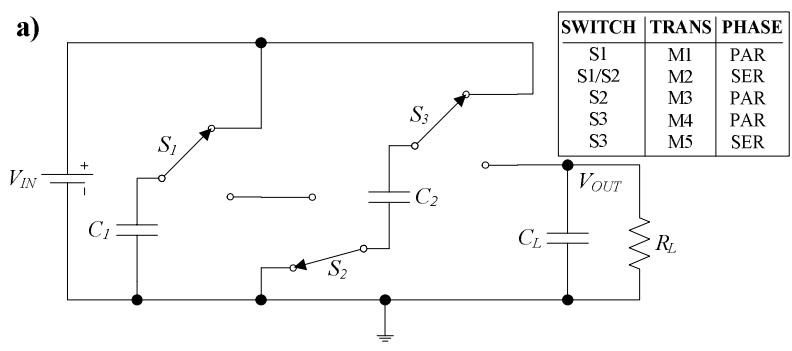

b)

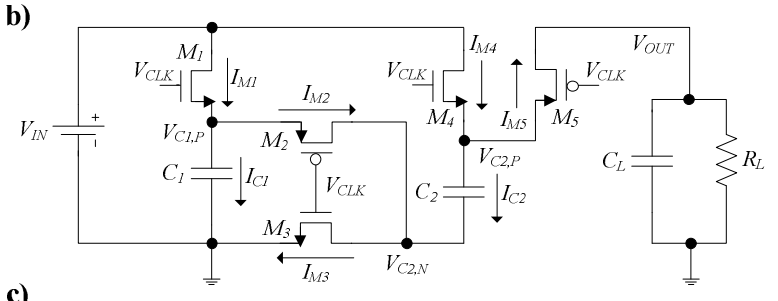

c)

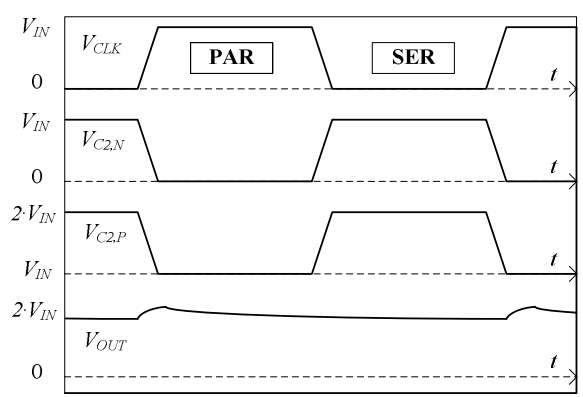

d)

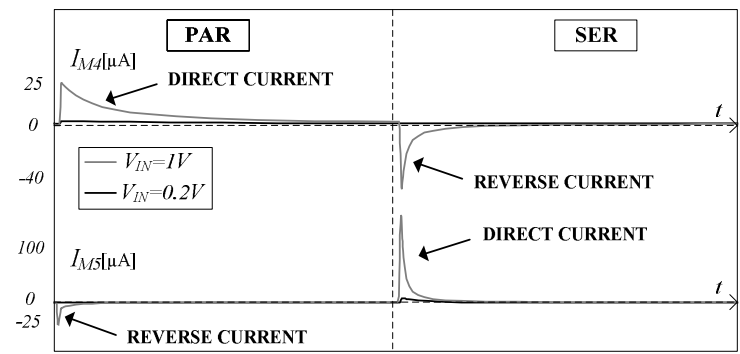

Fig 2. (a) Switched capacitor charge pump (SCCP). (b) Transistor-based implementation of the SCCP. (c) Ideal waveforms used to illustrate the operation of the SCCP. (d) Currents flowing through transistors $M_{4}$ and $M_{5}$ for $V_{I N}=0.2 \mathrm{~V}$ and $V_{I N}=1 \mathrm{~V}$.

transistors $M_{4}$ and $M_{5}$ are far beyond the elbow that marks the beginning of the reverse conduction zone (see Fig 1).

Reverse currents can be reduced if transistors $M_{4}$ and $M_{5}$ are not operated with reversed drain to source bias above the diode's on voltage. To achieve this it is necessary that these transistors in the original topology are implemented by the series connection of two transistors (i.e. $M_{4 A}$ and $M_{4 B}$ for $M_{4}$ and $M_{5 A}$ and $M_{5 B}$ for $M_{5}$ ), as shown in the marked area of Fig. 3a. Fig. 3b shows the currents through the transistors $M_{4}$ and $M_{5}$ of the original and the proposed topologies for 
a)

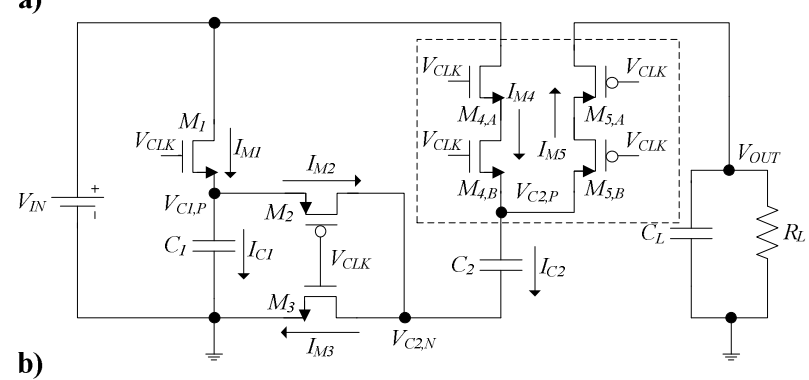

b)

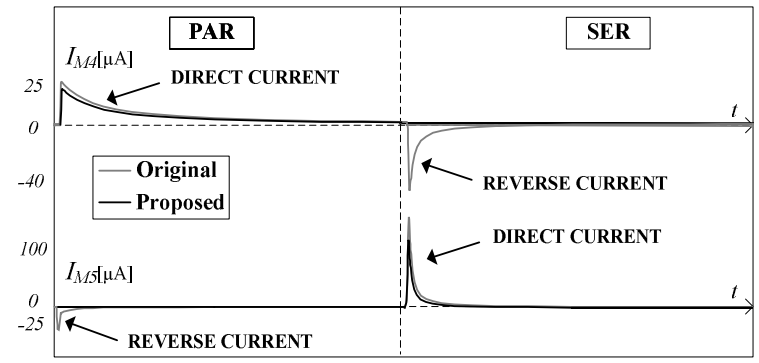

Fig. 3 (a) Proposed modification of the original SCCP to reduce the impact of reverse currents. (b) Currents flowing through transistors $M_{4}$ and $M_{5}\left(V_{I N}=1 \mathrm{~V}\right)$ for the original and the modified SCCP circuits.
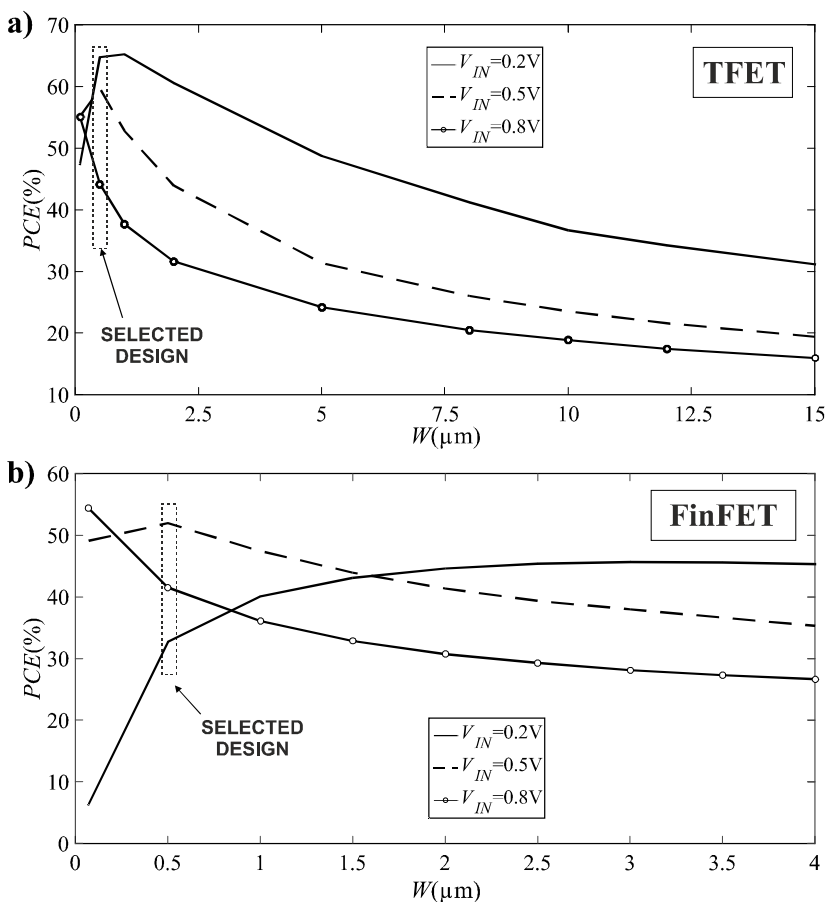

Fig. $4 P C E$ vs $W$ for (a) $1 P H$ (b) $2 P H-F i n F E T$.

$V_{I N}=1 \mathrm{~V}$. Note that the modified topology allows a significant reduction of reverse currents due to the distribution of the drain to source voltages between the series connected transistors.

\section{SimUlation RESUlTS}

The proposed modification of the original topology is evaluated using the TFET technology described in Section I. Simulation results are compared with those obtained for the original topology (hereafter " $1 P H "$ ). In addition, a modification of $1 P H$ employing two non-overlapping clock phases $\left(V_{C L K, 1}\right.$ and $\left.V_{C L K, 2}\right)$ is considered to avoid current leakage from $M_{2}$ to $M_{3}$ at transitions when the clock switches low. This topology (" $\left.2 P H^{\prime \prime}\right)$ applies $V_{C L K, 1}$ to the gate of all transistors except in $M_{2}$, which is replaced by an $N$ type transistor. When $V_{C L K, I}$ is at high level the $P A R$ phase is activated and the SER phase when $V_{C L K, 2}$ is. The $M_{5}$ transistor is not switched to $\mathrm{N}$ type to ensure that the increase in the output voltage does not affect its gate-source voltage. Moreover, being $\mathrm{P}$ type, it is activated when lowering $V_{C L K, 1}$, guaranteeing a suitable $V_{G S}$ voltage to operate properly. Both $1 P H$ and $2 P H$ topologies are evaluated using the proposed configuration (" $1 P H-P R O P$ " and " $2 P H-P R O P$ "). In addition, the $2 P H$ topology is designed using predictive 20nm FinFET transistors for high-performance (HP) obtained from the PTM web page [18] and is denoted as " $2 P H-F i n F E T$ '.

The input of the SCCP has been modelled as a DC voltage source that takes values between $50 \mathrm{mV}$ and $1 \mathrm{~V}$. Clock signals are pulse trains of a frequency of $10 \mathrm{MHz}$. This work aims to highlight the problem of the design of charge pumps using TFETs, evaluating and comparing the advantages of the proposed solution with respect to the conventional one. We are aware that a more comprehensive evaluation should include non-ideal clocks; however, the obtained results are fairly conclusive and should not vary significantly in a more realistic simulation environment.

In these experiments we consider that $C_{I}=C_{2}=1 \mathrm{pF}, C_{L}=1 \mathrm{pF}$ and $R_{L}=1 \mathrm{M} \Omega$ and the same transistor width $(W)$ for all devices. The selection of $W$ for both TFET and FinFET implementations is based on the evaluation of the power conversion efficiency $\left(P C E=P_{O U T} / P_{I N}\right)$ of $1 P H$ and $2 P H-$ FinFET for three values of the input voltage $(0.2 \mathrm{~V}, 0.5$ and $0.8 \mathrm{~V})$. For $1 P H, W=0.5 \mu \mathrm{m}$ has been selected because it gives an adequate trade-offs between $P C E$ at the three input voltage levels, as depicted in Fig. 4a. In 2PH-FinFET topology (Fig. $4 \mathrm{~b}$ ), $W=0.5 \mu \mathrm{m}$ has been chosen because it allows to obtain the almost the best $P C E$ values for $V_{I N}=0.5 \mathrm{~V}$ and $V_{I N}=0.8 \mathrm{~V}$ without significantly degrading the $P C E$ for $V_{I N}=0.8 \mathrm{~V}$.

Fig. 5a illustrates $P C E$ versus the range of input voltages analyzed for the five topologies studied. These results show that the TFET-based designs are more efficient than the one that uses FinFET transistors. On the other hand it is observed that, unlike conventional topologies, for the larger values of the input voltage efficiency hardly degrade due to reverse conduction currents. For example, for $V_{I N}=1 \mathrm{~V} P C E$ improves by approximately $25 \%$ with respect to the original $(1 P H$ and $2 P H)$ and FinFET topologies. Also note the improvements achieved in the proposed topology by using the two-phase clock scheme ( $10 \%$ for $\left.V_{I N}=1 \mathrm{~V}\right)$.

An alternative representation is shown in Fig. 5b, in which the efficiency is plotted versus the input power $\left(P_{I N}\right)$. Indeed, it can be observed that, for the larger input powers (associated with large input voltages), the efficiency of the proposed topologies is better. The impact of the series connection of the two transistors in the proposed solution is manifested in the slight reduction of $P C E$ achieved for the lower values of the input voltage (power).

The modification of the proposed topology can be easily extended to more complex (and efficient) charge pumps designs in which reverse currents are obtained. In the TFET-based cross coupled implementation of a charge pump [16], this problem occurs again for high values of $V_{I N}$. Similarly to the $S C C P$ topology, the proposed modification based on the series connection of transistors reduces the impact of these reverse currents, improving their power conversion efficiency. Fig. 6a shows $P C E$ versus the $V_{I N}$ for the original and the proposed topologies, verifying that, 

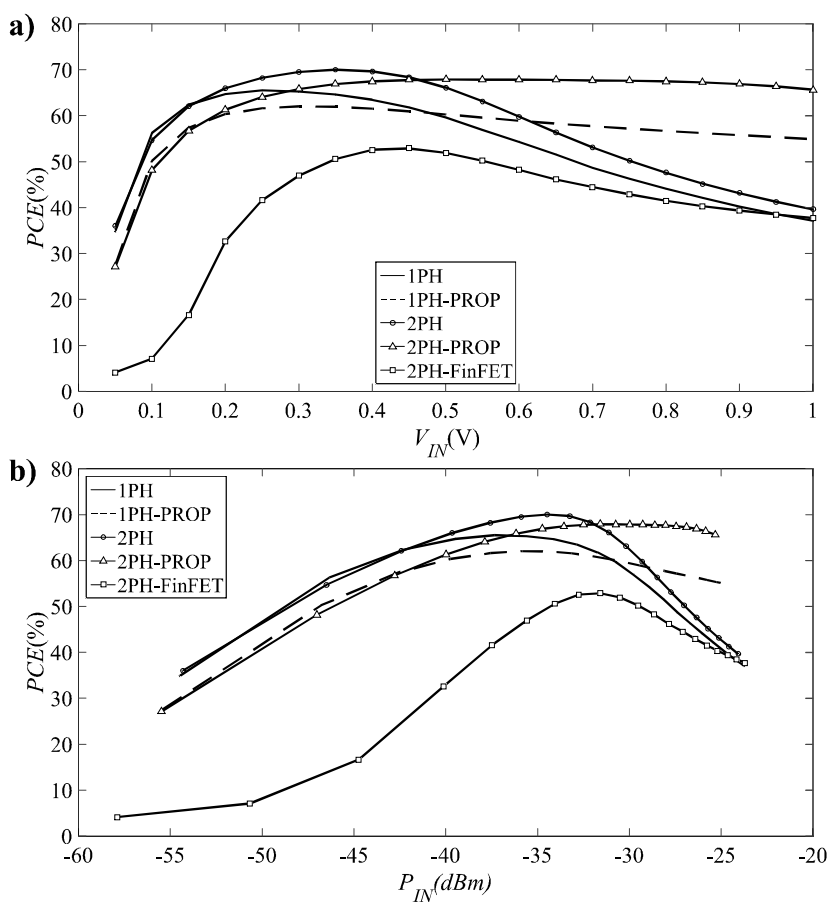

Fig. 5 Evaluation of performances of original and proposed implementations of the $S C C P$ using TFETs and FinFETs. (a) $P C E$ vs $V_{I N}$, (b) $P C E$ vs $P_{I N}$.
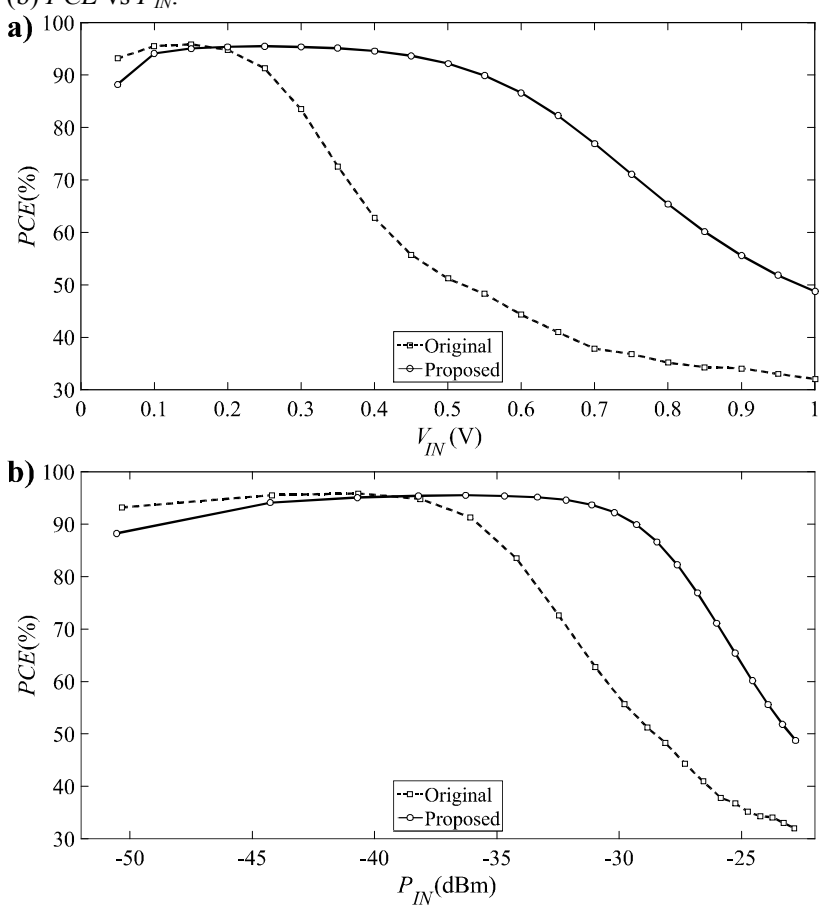

Fig. 6 Evaluation of the performances of the original and proposed cross coupled charge pumps. (a) $P C E$ vs $V_{I N}$, (b) $P C E$ vs $P_{I N}$.

from $\quad V_{I N}=0.2 \mathrm{~V}, \quad$ approximately $P C E_{P R O P}\left(2 \cdot V_{I N}\right)=$ $P C E_{\text {ORIG }}\left(V_{I N}\right)$ is satisfied. $P C E$ versus the input power is depicted in Fig. 6b, where it can be observed that efficiencies above $90 \%$ up to $-37 \mathrm{dBm}$ are obtained for the original topology whereas for the proposed they are achieved up to $-30 \mathrm{dBm}(1 \mu \mathrm{W})$.

\section{CONCLUSIONS}

The design of charge pumps using tunnel transistors must take into account the existence of reverse conduction currents due to direct polarization of the $p-i$ - $n$ diodes. In this paper we propose a modification of a conventional charge pump topology, based on the serial connection of certain transistors that reduces the impact of these currents and, therefore, improves its power conversion efficiency.

\section{ACKNOWLEDGEMENT}

This work has been funded by Ministerio de Economía y Competitividad del Gobierno de España with support from FEDER (Projects TEC2013-40670-P, TEC2017-87052-P and TEC2013-45638-C3-3-R).

\section{REFERENCES}

[1] X. Li, K. Ma, S. George, J. Sampson and V. Narayanan, "Enabling Internet-of-Things: Opportunities brought by emerging devices, circuits, and architectures," 2016 IFIP/IEEE International Conference on Very Large Scale Integration (VLSI-SoC), Tallinn, 2016, pp. 1-6.

[2] A. Seabaugh and Q. Zhang, "Low-voltage tunnel transistors for beyond CMOS logic," Proceedings of the IEEE, vol. 98, no. 12, Dec. 2010.

[3] Nikonov, Dmitri E., and Ian A. Young. "Benchmarking of beyondCMOS exploratory devices for logic integrated circuits." IEEE Journal on Exploratory Solid-State Computational Devices and Circuits, vol. 1 pp. 3-11, Dec. 2015.

[4] A.M. Ionescu, H. Riel: "Tunnel field-effect transistors as energyefficient electronic switches", Nature, no. 479, pp. 329-337, 2011.

[5] H. Lu T. Ytterdal, A. Seabaugh, "Universal TFET model". nanoHUB. doi:10.4231/D3901ZG9H.

[6] nanoHUB. https:// nanohub.org.

[7] H. Liu, S. Datta, V. Narayanan, "Steep switching tunnel FET: a promise to extend energy efficient roadmap for post-CMOS digital and analog/RF applications", Symp. on Low Power and Design, 2013.

[8] U. E. Avci, D.H. Morris and I.A. Young, "Tunnel Field-Effect Transistors: Prospect and Challenges”, IEEE Journal of the Electron Device Society, vol. 3, no. 3, pp. 88-95, Jan. 2015.

[9] J. Núñez, M.J. Avedillo, “J. Núñez and M. J. Avedillo, "Comparison of TFETs and CMOS Using Optimal Design Points for Power-Speed Tradeoffs," in IEEE Transactions on Nanotechnology, vol. 16, no. 1, pp. 83-89, Jan. 2017.

[10] X. Li, U. Dennis Heo, K. Ma, V. Narayanan, H. Liu and S. Datta, "RF-powered systems using steep-slope devices," 2014 IEEE 12th International New Circuits and Systems Conference (NEWCAS), Trois-Rivieres, QC, 2014, pp. 73-76.

[11] D. Cavalheiro, F. Moll and S. Valtchev, "TFET-Based Power Management Circuit for RF Energy Harvesting," in IEEE Journal of the Electron Devices Society, vol. 5, no. 1, pp. 7-17, Jan. 2017.

[12] H. Liu, X. Li, R. Vaddi, K. Ma, S. Datta and V. Narayanan, "Tunnel FET RF Rectifier Design for Energy Harvesting Applications," in IEEE Journal on Emerging and Selected Topics in Circuits and Systems, vol. 4, no. 4, pp. 400-411, Dec. 2014.

[13] J. Núñez and M. J. Avedillo, "Reducing the Impact of Reverse Currents in Tunnel FET Rectifiers for Energy Harvesting Applications," IEEE Journal of the Electron Devices Society, vol. PP, no. 99, pp. 1-1, Aug. 2017.

[14] D. Cavalheiro, F. Moll, S. Valtchev, "Insights into tunnel FET-based charge pumps and rectifiers for energy harvesting applications," IEEE Transactions on Very Large Scale Integration (VLSI) Systems, vol. 25, no. 3, pp. 988-997, Mar. 2017.

[15] D. Cavalheiro, F. Moll and S. Valtchev, "Pespectives of TFET devices in ultra-low power charge pumps for thermo-electric energy sources," 2015 IEEE International Symposium on Circuits and Systems (ISCAS), Lisbon, 2015, pp. 1082-1085.

[16] U. Heo, X. Li, H. Liu, S. Gupta, S. Datta and V. Narayanan, "A High-Efficiency Switched-Capacitance HTFET Charge Pump for Low-Input-Voltage Applications," 28th International Conference on VLSI Design, Bangalore, 2015, pp. 304-309.

[17] J. S. Brugler, "Theoretical performance of voltage multiplier circuits," IEEE J. Solid State Circuits, vol. SSC-6, no. 3, pp. 132135, June 1971.

[18] W. Zhao and Y. Cao, "New generation of predictive technology model for sub-45nm design exploration", Proc. $7^{\text {th }}$ Int. Symp. Quality Electronic Design, 2006. 